\title{
Possible importation and subsequent cross-transmission of OXA-48-producing Klebsiella pneumoniae, France, 2010
}

D Decre (dominique.decre@sat.aphp.fr) ${ }^{1,2}$, G Birgand ${ }^{3}$, D Geneste ${ }^{2}$, E Maury ${ }^{4}$, J C Petit ${ }^{1}$, F Barbut ${ }^{3}$, G Arlet ${ }^{2}$

1. Assistance Publique-Hôpitaux de Paris, Microbiology, Saint-Antoine Hospital, Paris, France

2. University Pierre and Marie Curie, ER8 Antibiotiques et Flore digestive, Paris VI, France

3. Assistance Publique-Hôpitaux de Paris, Unité d’Hygiène et de Lutte contre les Infections Nosocomiales, Saint-Antoine Hospital, Paris, France

4. Assistance Publique-Hôpitaux de Paris, Medical Intensive Care Unit, Saint-Antoine Hospital, Paris, France

Decre D, Birgand G, Geneste D, Maury E, Petit JC, Barbut F, Arlet G. Possible importation and subsequent cross-transmission of OXA-48-producing Klebsiella pneumoniae, France, 2010. Euro Surveill. 2010;15(46):pii=19718. Available online: http://www.eurosurveillance.org/ViewArticle.aspx?Articleld=19718

Article published on 18 November 2010

We report the possible first patient-to-patient transmission of Klebsiella pneumoniae with decreased susceptibility to imipenem and producing OXA-48, CTX-M15, TEM-1 and OXA-1 in a French hospital.

\section{Background}

The class D beta-lactamase OXA-48 conferring decreased susceptibility to carbapenems in Klebsiella pneumoniae was firstly identified in sporadic isolates from Turkey. Subsequently, large outbreaks have been described in Istanbul and other cities in Turkey [1]. OXA48-producing $K$. pneumoniae isolates have since been reported from the United Kingdom, Israel, Lebanon, Argentina, France, and recently from Germany, Tunisia and Morocco [2-6]. It has been shown that blaOXA-48 was located on Tn1999, a composite transposon made of two copies of IS1999 [2].

We report the possible first occurrence of patientto-patient transmission of OXA-48-producing K. pneumoniae in a French hospital following the likely importation of the index isolate from Morocco.

\section{Case 1}

The index patient lived in Morocco most of the year, who had an underlying chronic condition and had been hospitalised for an operation in Morocco in March 2009. The patient had been in France for a few weeks when, after having fallen, was admitted to a University hospital in Paris, in early March 2010. The case was hospitalised and underwent surgery on the day following admission. Almost one week later, the patient was transferred to the medical intensive care unit (ICU) for an acute respiratory failure. In this ICU, screening of patients for faecal carriage of extended-spectrumbeta-lactamase (ESBL)-producing Enterobacteriaceae is implemented since September 2009 and must be performed at admission and weekly.

\section{Laboratory results}

An ESBL-producing $K$. pneumoniae isolate was recovered from the rectal swab sampled from this patient at admission in the ICU. By using the disc diffusion method on Mueller-Hinton agar, the isolate showed resistance to fluoroquinolones, tobramycin, gentamicin and cotrimoxazole, and intermediate resistance level to imipenem. It has been determined through the same method that the isolate was only susceptible to colistin and amikacin. The zone diameter for imipenem was of $20 \mathrm{~mm}$ and the minimum inhibitory concentration (MIC) determined by Etest (Bio-Rad Laboratoires) was $2 \mathrm{mg} / \mathrm{L}$. The modified Hodge test was positive. PCR using a panel of primers specific for the detection of Klebsiella pneumoniae carbapenemase (KPC) acquired AmpC-type beta-lactamases, OXA-type carbapenemases, metallo-beta-lactamases (MBLs) and ESBLs [7] and sequencing revealed the presence of bla $a_{\mathrm{TEM}-1}$, bla $a_{\mathrm{OXA}-1}, b_{\mathrm{TX}-\mathrm{M}-15}$ and bla $\mathrm{OXX}_{\mathrm{OX}-48}$ beta-lactamase genes.

\section{Control measures}

On 19 March, barrier precautions were implemented, and promotion of hand hygiene using alcohol-based products and room cleaning were reinforced. In addition, screening for faecal carriage of OXA-48-producing $K$. pneumoniae was performed. Rectal swabs were recovered from all patients who had been hospitalised in the medical ICU or in the orthopaedic ward during the same period as the index patient.

\section{Case 2}

At the end of March 2010, a second patient colonised with OXA-48 producing $K$. pneumoniae was notified by the laboratory. This second patient had initially been admitted in the medical ICU with pneumonia. Upon admission, following screening, the case was found negative for ESBL and then transferred to the geriatric ward where weekly screening was continued. The epidemiological analysis showed that this patient had stayed in the same area of the medical ICU as the index 
patient for six days, and that both patients had been managed by the same team of healthcare workers, suggesting potential cross-transmission. Both patients had previously been receiving broad-spectrum antibiotics such as third generation cephalosporins but not carbapenems.

\section{Laboratory results}

The two isolates showed the same resistance phenotype to beta-lactams, aminoglycosides and quinolones. Enterobacterial repetitive intergenic consensus (ERIC). PCR analysis showed the same profile compared with non-epidemiologically related $K$. pneumoniae strains (data not shown). As reported for the strain isolated in Tunisia [5], plasmid analysis indicated the presence of three plasmids and the plasmid of approximatively 70-80 Kb carried bla $a_{0 \times A-48^{\circ}}$.

\section{Control measures}

Following identification of OXA-48-producing K. pneumoniae in the second patient, a set of infection control measures were implemented. This set included (i) cohorting of the two colonised patients in the geriatric ward, (ii) stopping transfers of the two colonised patients and of patients who had previously been in contact with them, (iii) performing a systematic search for additional patients who could have been in contact with the colonised patients, (iv) screening of all contact patients by rectal swabbing once a week, or twice a week for contact patients receiving antibiotics. No other OXA-48-producing- $K$. pneumoniae has been isolated in the hospital since 31 March 2010.

\section{Discussion and conclusion}

The global spread of ESBLs, particularly CTX-M enzymes, in clinical isolates of Escherichia coli and $K$. pneumoniae has driven therapeutic choice towards carbapenems and lead to emergence of carbapenem resistance mechanisms. Carbapenemases now represent a public health challenge. In Enterobacteriaceae, carbapenemases are diverse; these belong to betalactamases molecular class A (KPC), class B (IMP, VIM) and class D (OXA-48), are involved in outbreaks in various geographical regions and are increasingly reported in sporadic cases worldwide $[8,9]$. Until recently, OXA48 seemed to be limited to few countries, but has begun to spread, in particular in countries from the eastern and southern Mediterranean region $[2,5,6]$. In Germany, OXA-48 is the most frequent carbapenemase [5]. Since 2005, all beta-lactamases from multidrug. resistant Enterobacteriaceae isolates in our institution have been characterised and this is the first strain identified as producing OXA-48. The Moroccan origin of the strain is suggested by the fact that the index patient mostly lived in Morocco where OXA-48 has recently been reported [6], that she had not previously been hospitalised in France and that none of the contact patients from the orthopaedic ward was colonised.

Since OXA-48 remains difficult to detect, especially when it is not associated with an ESBL [10], enhanced surveillance and rapid identification are essential. In addition, once OXA-48 is identified, adequate infection control measures should rapidly be implemented to prevent cross-transmission.

Acknowledgements

We are grateful to Bruno Coignard (Institut de Veille Sanitaire) for helpful discussion.

\section{References}

1. Carrër A, Poirel L, Eraksoy H, Cagatay AA, Badur S, Nordmann P. Spread of OXA-48-positive-carbapenem-resistant Klebsiella pneumoniae isolates in Istanbul, Turkey. Antimicrob Agents Chemother. 2008;52(8):2950-4.

2. Carrër A, Poirel L, Yilmaz M, Akan OA, Feriha C, Cuzon G, et al. Spread of OXA-48 encoding plasmid in Turkey and beyond. Antimicrob Agents Chemother. 2010;54(3):1369-73.

3. Nordmann P, Cuzon G, Naas T. The real threat of Klebsiella pneumoniae carbapenemase producing bacteria. Lancet Infect Dis. 2009;9(4):228-36.

4. Grundmann H, Livermore DM, Giske CG, Canton R, Rossolini GM, Campos J, et al. Carbapenem-non-susceptible Enterobacteriaceae in Europe: conclusions from a meeting of national experts. Euro Surveill. 2010;15(46): $\mathrm{pii}=19711$. Available from: http://www.eurosurveillance.org/ViewArticle. aspx?Articleld $=19711$

5. Cuzon G, Naas T, Lesenne A, Benhamou M, Nordmann P. Plasmid-mediated carbapenem-hydrolysing OXA-48 口-lactamase in Klebsiella pneumoniae from Tunisia. Int J Antimicrob Agents. 2010;36(1):91-3.

6. Benouda A, Touzani O, Khairallah MT, Araj GF, Matar GM First detection of an oxacillinase-mediated resistance to carbapenem in Klebsiella pneumonia from Morroco. Ann Trop Med Parasitol. 2010;104(4):327-30.

7. Dallenne C, Da Costa A, Decré D, Favier C, Arlet G. Development of a set of multiplex PCR assays for the detection of genes encoding important $\beta$-lactamases in Enterobacteriaceae. J Antimicrob Chemother. 2010;65(3):490-5.

8. Kassis-Chikhani N, Decré D, Gautier V, Burghoffer B, Saliba F, Mathieu D, et al. First outbreak of multidrug-resistant Klebsiella pneumoniae carrying blaVIM-1 and blaSHV-5 in a French University hospital. J Antimicrob Chemother. 2006;57(1):142-5.

9. Kassis-Chikhani N, Decré D, Ichai P, Sengelin C, Geneste $D$, Mihaila L, et al. Outbreak of Klebsiella pneumoniae producing KPC-2 and SHV-12 in a French hospital. J Antimicrob Chemother. 2010;65(7):1539-40.

10. Cuzon G, Naas T, Bogaerts P, Glupczynski Y, Huang TD, Nordmann P. Plasmid-encoded carbapenem-hydrolizing betalactamase OXA-48 in an imipenem-susceptible Klebsiella pneumoniae strain from Belgium. Antimicrob Agents Chemother. 2008;52(9):3463-4. 